\title{
Advanced Modeled Iterative Reconstruction in Low-Tube-Voltage Contrast-Enhanced Neck CT: Evaluation of Objective and Subjective Image Quality
}

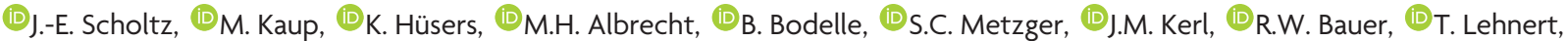

(1)T.J. Vogl, and (1).L. Wichmann

\begin{abstract}
BACKGROUND AND PURPOSE: Dose-saving techniques in neck CT cause increased image noise that can be counteracted by iterative reconstruction. Our aim was to evaluate the image quality of advanced modeled iterative reconstruction (ADMIRE) in contrast-enhanced low-tube-voltage neck CT.

MATERIALS AND METHODS: Sixty-one patients underwent 90-kV(peak) neck CT by using third-generation 192-section dual-source CT. Image series were reconstructed with standard filtered back-projection and ADMIRE strength levels 1, 3, and 5. Attenuation and noise of the sternocleidomastoid muscle, internal jugular vein, submandibular gland, tongue, subscapularis muscle, and cervical fat were measured. Signal-to-noise and contrast-to-noise ratios were calculated. Two radiologists assessed image noise, image contrast, delineation of smaller structures, and overall diagnostic acceptability. Interobserver agreement was calculated.

RESULTS: Image noise was significantly reduced by using ADMIRE compared with filtered back-projection with the lowest noise observed in ADMIRE 5 (filtered back-projection, $9.4 \pm 2.4$ Hounsfield units [HU]; ADMIRE 1, $8.3 \pm 2.8 \mathrm{HU}$; ADMIRE 3, $6.7 \pm 2.0$ HU; ADMIRE 5, $5.4 \pm$ $1.7 \mathrm{HU}$; all, $P<.001)$. Sternocleidomastoid SNR and internal jugular vein-sternocleidomastoid contrast-to-noise ratios were significantly higher for ADMIRE with the best results in ADMIRE 5 (all, $P<.001$ ). Subjective image quality and image contrast of ADMIRE 3 and 5 were consistently rated better than those for filtered back-projection and ADMIRE 1 (all, $P<.001$ ). Image noise was rated highest for ADMIRE 5 (all, $P<.005)$. Delineation of smaller structures was voted higher in all ADMIRE strength levels compared with filtered back-projection $(P<$ .001). Global interobserver agreement was good (0.75).
\end{abstract}

CONCLUSIONS: Contrast-enhanced 90-kVp neck CT is feasible, and ADMIRE 5 shows superior objective image quality compared with filtered back-projection. ADMIRE 3 and 5 show the best subjective image quality.

ABBREVIATIONS: ADMIRE = advanced modeled iterative reconstruction; $\mathrm{CNR}=$ contrast-to-noise ratio; $\mathrm{DSCT}=$ dual-source CT; $\mathrm{FBP}=$ filtered back-projection; $\mathrm{HU}=$ Hounsfield units

C ontrast-enhanced CT is a well-established initial cross-sectional imaging technique for examination of the head and neck region. ${ }^{1-3}$ Several strategies have been developed for both radiation dose reduction and improvement of image quality. These typically involve adjusting CT acquisition parameters such as tube voltage, tube current, tube rotation time, pitch, and colli-

Received May 4, 2015; accepted after revision June 10.

From the Department of Diagnostic and Interventional Radiology (J.-E.S., M.K., K.H., M.H.A., B.B., S.C.M., J.M.K., R.W.B., T.L., T.J.V., J.L.W.), University Hospital Frankfurt, Frankfurt, Germany; and Department of Radiology and Radiological Science (J.L.W.), Medical University of South Carolina, Charleston, South Carolina. Jan-Erik Scholtz and Moritz Kaup contributed equally and share first authorship. Neither Ralf W. Bauer nor J. Matthias Kerl analyzed or controlled any data in this study.

Please address correspondence to Jan-Erik Scholtz, MD, Universitätsklinikum Frankfurt am Main, Institut für Diagnostische und Interventionelle Radiologie, TheodorStern-Kai 7, 60590 Frankfurt; e-mail: janerikscholtz@gmail.com

http://dx.doi.org/10.3174/ajnr.A4502 mation to the patient body and examined body region. ${ }^{4-6}$ The interaction of these parameters is complex, and manual adjustments may result in nondiagnostic images. Thus, commercially available techniques, including tube current modulation, ${ }^{7}$ automatic exposure control, ${ }^{8,9}$ automated tube voltage adaptation, ${ }^{10,11}$ iterative reconstruction, ${ }^{12-15}$ and selective in-plane shielding (thyroid, eye lens, breast, and gonads), ${ }^{16}$ have been introduced to support the radiologic technologist, physicist, and radiologist team in developing appropriate CT protocols.

Reduced tube voltage can increase contrast-to-noise ratio (CNR) of iodine enhancing soft-tissue structures, while the radiation dose is substantially reduced. ${ }^{4}$ The drawback of an increased image noise in low-tube-voltage examinations can be counteracted by iterative reconstruction, which reduces image noise compared with filtered back-projection (FBP). ${ }^{12,14}$ Recently introduced advanced modeled iterative reconstruction (ADMIRE) performs detailed modeling in the projection data domain, result- 
Table 1: Results of objective image analysis ${ }^{a}$

\begin{tabular}{lrrrr}
\hline & \multicolumn{1}{c}{ FBP } & ADMIRE 1 & \multicolumn{1}{c}{ ADMIRE 3 } & \multicolumn{1}{c}{ ADMIRE 5 } \\
\hline Attenuation (HU) & & & & \\
$\quad$ Sternocleidomastoid muscle & $79.4 \pm 13.2$ & $80.1 \pm 13.4$ & $79.9 \pm 13.6$ & $79.2 \pm 13.7$ \\
$\quad$ Internal jugular vein & $288.5 \pm 71.4$ & $290.5 \pm 72.6$ & $289.0 \pm 71.6$ & $287.3 \pm 72.2$ \\
$\quad$ Submandibular gland & $131.4 \pm 47.1$ & $131.2 \pm 48.2$ & $131.6 \pm 47.8$ & $131.1 \pm 47.2$ \\
$\quad$ Tongue & $97.4 \pm 14.1$ & $97.5 \pm 13.9$ & $96.2 \pm 13.5$ & $95.7 \pm 13.5$ \\
Subscapularis muscle & $69.8 \pm 12.9$ & $69.9 \pm 12.8$ & $68.8 \pm 12.6$ & $69.0 \pm 12.4$ \\
$\quad$ Fat & $-105.7 \pm 9.3$ & $-106.4 \pm 11.3$ & $-107.2 \pm 10.9$ & $-106.0 \pm 11.3$ \\
Image noise (HU) & & & & \\
$\quad$ Sternocleidomastoid muscle & $9.4 \pm 2.4$ & $8.3 \pm 2.8$ & $6.7 \pm 2.0$ & $5.4 \pm 1.7$ \\
$\quad$ Internal jugular vein & $12.6 \pm 6.3$ & $11.2 \pm 5.9$ & $10.3 \pm 7.1$ & $8.5 \pm 5.5$ \\
Submandibular gland & $11.9 \pm 2.6$ & $11.2 \pm 2.5$ & $9.4 \pm 2.9$ & $7.5 \pm 3.0$ \\
$\quad$ Tongue & $10.3 \pm 2.4$ & $9.8 \pm 2.6$ & $8.6 \pm 2.8$ & $6.9 \pm 3.2$ \\
$\quad$ Subscapularis muscle & $15.3 \pm 2.8$ & $13.7 \pm 2.7$ & $11.8 \pm 2.6$ & $8.6 \pm 2.8$ \\
$\quad$ Fat & $15.1 \pm 5.1$ & $14.9 \pm 4.5$ & $12.6 \pm 4.8$ & $9.8 \pm 4.0$ \\
\hline at & & & &
\end{tabular}

ing in less noise and improved artifact suppression. ${ }^{17}$ ADMIRE includes a local signal-to-noise relationship analysis and decomposes the image data into information and noise. ${ }^{18}$ Further technical details have been described in recent studies. ${ }^{14,17,18}$ Thus, neck CT may potentially be performed with a reduced tube voltage and therefore lower radiation dose without impairing image quality.

The purpose of our study was to evaluate the impact of ADMIRE on image quality in low-tube-voltage contrast-enhanced neck CT compared with FBP on a 192-section third-generation dual-source CT (DSCT).

\section{MATERIALS AND METHODS Patient Population}

This retrospective study was approved by the ethics committee of our hospital, and the requirement for written informed consent was waived. Sixty-four patients (54.6 \pm 16.4 years of age; range, 24-82 years) underwent contrast-enhanced neck CT between November 2014 and February 2015. These time intervals were chosen due to a change in tube voltage to $90 \mathrm{kV}$ in the standard protocol for contrast-enhanced neck CT in adults on the DSCT scanner used in October 2014. Our study population consisted of 38 males ( $58.1 \pm 16.1$ years of age; range, $24-82$ years) and 26 females ( $49.5 \pm 16.1$ years of age; range, $26-75$ years). Indications for contrast-enhanced neck CT included detection or exclusion $(n=26)$ or follow-up $(n=10)$ of a tumor or lymphoma in the head and neck region or the visualization or exclusion of a clinically suspected inflammatory process $(n=28)$.

CT angiography and noncontrast examinations were excluded from this study. Furthermore, nondiagnostic studies due to severe motion or metal artifacts were excluded. Underage patients (younger than 18 years of age) were excluded because they are examined with different scan protocols at our institution. Contraindications for CT imaging were any known previous reactions to iodinated contrast medium, renal impairment with a glomerular filtration rate lower than $60 \mathrm{~mL} / \mathrm{min}$, and known pregnancy.

\section{Examination Protocol}

All examinations were performed on a 192-section third-generation DSCT (Somatom Force; Siemens, Erlangen, Germany) in single-energy mode. The examinations were planned according to flow rate of $2 \mathrm{~mL} / \mathrm{s}$.

\section{CT Data Reconstruction}

Image series were reconstructed in axial views with a section thickness of $2 \mathrm{~mm}$ (2-mm increment) by using an FBP algorithm with a soft-tissue convolution kernel (B30f) and ADMIRE with a soft-tissue convolution kernel (Br30f). The technical features of ADMIRE have been described previously. ${ }^{14,19}$ ADMIRE provides 5 strength levels (1-5); each examination was reconstructed in strength levels 1,3 , and 5 . We chose these reconstruction levels because level 3 is recommended as the standard for most contrastenhanced CT examinations by the vendor, and we could compare the impact of image reconstruction, with very little ADMIRE contribution (level 1) and maximum ADMIRE influence (level 5), with the standard. We omitted the levels in-between (levels 2 and 4) because we assumed that differences in image quality may be less apparent. Images were series reconstructed in clinical routine with an average reconstruction time of $0.5-1$ minute for each parameter without differences in time required among the different ADMIRE levels.

\section{Objective Image Analysis}

All measurements were performed on a commercially available PACS workstation. We evaluated the following anatomic structures: the sternocleidomastoid muscle, internal jugular vein, tongue, submandibular gland, cervical fat, and the subscapularis muscle. Circular ROIs (10-30 $\left.\mathrm{mm}^{2}\right)$ were drawn as large as possible in these structures while carefully avoiding inclusion of adjacent anatomic structures or focal regions of inhomogeneity. Signal attenuation and image noise were measured in Hounsfield units (HU). Values averaged of 3 measurements were calculated to ensure data consistency. Image noise was quantified as the SD of each measured anatomic structure. Signal-to-noise ratio was calculated for the sternocleidomastoid muscle and submandibular gland by using the following formula:

$$
\mathrm{SNR}=\frac{\text { Mean Signal Intensity }}{\mathrm{SD}}
$$

The sternocleidomastoid muscle-fat contrast-to-noise ratio and submandibular gland-fat CNR were calculated as follows: 


$$
\mathrm{CNR}=\frac{\mathrm{HU}(\text { Soft-Tissue Structure })-\mathrm{HU}(\text { Fat })}{\text { Image Noise }(\text { Fat })} .
$$

The internal jugular vein (IJV)-sternocleidomastoid muscle CNR was calculated as follows:

$\mathrm{CNR}=\frac{\text { Attenuation (IJV) }- \text { Attenuation (Sternocleidomastoid Muscle) }}{\text { Image Noise (Sternocleidomastoid Muscle) }}$.

\section{Subjective Image Analysis}

Patients were evaluated in a randomized manner independently by 2 radiologists with 3 and 4 years of experience in neck CT, respectively. Observers were blinded to the reconstruction technique used. All CT images were preadjusted to the same soft-tissue

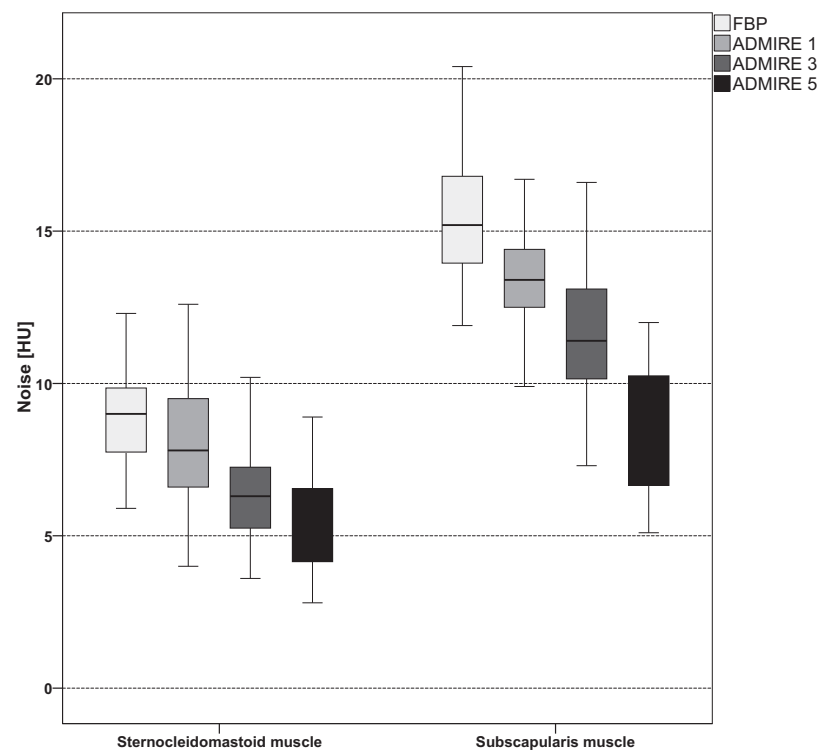

FIG 1. Boxplot graph shows comparison of image noise of the sternocleidomastoid and subscapularis muscles between the different image reconstruction settings. Image noise of the sternocleidomastoid muscle and subscapularis muscle was significantly lower with all ADMIRE levels, with the best results for ADMIRE 5 (all, $P<.001$ ). Noise in the lower part of the neck, represented by the subscapularis muscle, was significantly increased within each reconstruction mode compared with the upper part of the neck, represented by the sternocleidomastoid muscle (all, $P<.001$ ).

Table 2: Signal-to-noise ratio and contrast-to-noise ratio calculations ${ }^{a}$

\begin{tabular}{lcccc}
\hline & FBP & ADMIRE 1 & ADMIRE 3 & ADMIRE 5 \\
\hline $\begin{array}{l}\text { Signal-to-noise ratio } \\
\quad \text { Sternocleidomastoid muscle }\end{array}$ & $9.0 \pm 2.5$ & $10.8 \pm 4.1$ & $13.0 \pm 4.4$ & $16.4 \pm 6.2$ \\
$\quad$ Submandibular gland & $11.6 \pm 4.6$ & $12.3 \pm 4.7$ & $15.3 \pm 6.8$ & $19.6 \pm 8.2$ \\
$\quad \begin{array}{l}\text { Contrast-to-noise ratio } \\
\quad \text { Sternocleidomastoid muscle-fat }\end{array}$ & $14.5 \pm 8.1$ & $13.8 \pm 4.9$ & $17.3 \pm 7.7$ & $22.6 \pm 11.4$ \\
$\quad$ Submandibular gland-fat & $19.1 \pm 11.6$ & $17.9 \pm 8.2$ & $22.6 \pm 11.7$ & $28.6 \pm 17.1$ \\
$\quad$ IJV-sternocleidomastoid muscle & $24.1 \pm 10.1$ & $28.7 \pm 13.5$ & $34.8 \pm 16.3$ & $41.2 \pm 22.1$ \\
\hline
\end{tabular}

Note:-IJV indicates internal jugular vein.

${ }^{a}$ Data are means.

Table 3: Subjective image-quality assessment ${ }^{\mathrm{a}}$

\begin{tabular}{lcccc}
\hline & FBP & ADMIRE 1 & ADMIRE 3 & ADMIRE 5 \\
\hline Overall image quality & $3.2 \pm 0.5(0.58)$ & $3.3 \pm 0.6(0.57)$ & $4.4 \pm 0.9(0.12)$ & $4.7 \pm 0.5(0.67)$ \\
Image noise & $3.4 \pm 0.5(0.79)$ & $3.8 \pm 0.4(0.83)$ & $4.6 \pm 0.5(0.52)$ & $4.9 \pm 0.3(0.32)$ \\
Delineation of smaller & $3.3 \pm 0.4(0.74)$ & $3.7 \pm 0.5(0.78)$ & $3.8 \pm 0.4(0.50)$ & $3.8 \pm 0.4(0.74)$ \\
$\quad$ structures & & & & \\
Image contrast & $3.5 \pm 0.5(0.80)$ & $3.4 \pm 0.5(0.78)$ & $4.8 \pm 0.4(0.34)$ & $4.8 \pm 0.4(0.47)$ \\
\hline
\end{tabular}

${ }^{a}$ Data are means \pm SD. Interobserver agreement (slight $[\kappa<0.3]$, moderate $[\kappa=0.3-0.7]$, good [ $\left.\kappa>0.7\right]$ ). window setting (width, 400; level, 60). Reviewers were allowed to scroll through the complete presented axial image series with the possibility of freely adjusting the window width and level. Subjective image analyses were performed on rating scales of $1-5$ for overall image quality $(5=$ excellent, $4=$ good, $3=$ sufficient, $2=$ poor, $1=$ nondiagnostic); delineation of small structures of the pharynx (wall, mucosal margin, parapharyngeal fat, parapharyngeal muscle), larynx (mucosal folds, intrinsic laryngeal muscles, paralaryngeal muscles), and salivary glands (glandular tissue, paraglandular fat spaces); delineation of lymphatic tissue of Waldeyer tonsillar ring and cervical lymph ( $5=$ excellent visibility, $4=$ above average, $3=$ acceptable, $2=$ suboptimal, $1=$ very poor $)$; image contrast $(5=$ excellent image contrast, $4=$ above average, $3=$ acceptable, $2=$ suboptimal, $1=$ very poor); and image noise ( $5=$ very low, $4=$ low, $3=$ average, $2=$ considerable, 1 = high).

\section{Radiation Dose}

Radiation exposure was expressed as CT dose index volume $\left(\mathrm{CTDI}_{\mathrm{vol}}\right)$ and dose-length product, and it was provided automatically by the CT scanner. The estimated effective dose was calculated by using a standard conversion factor of 0.0051 for $100-\mathrm{kV}$ neck CT. In addition, size-specific dose estimates were calculated. To calculate specific dose estimates, we measured the effective diameter from the anteroposterior (AP) and lateral (LAT) dimensions at the fourth cervical vertebra.

$$
\text { Effective Diameter }(\mathrm{cm})=\sqrt{\mathrm{AP} \times \mathrm{LAT}} \text {. }
$$

A conversion factor based on the effective diameter and the $32-\mathrm{cm}$ diameter polymethylmethacrylate phantom provided by the American Association of Physicists in Medicine Report No. 204 was selected for each patient. ${ }^{20}$ Size-specific dose estimate (SSDE) was calculated as follows:

$$
\operatorname{SSDE}(\mathrm{mGy})=\mathrm{CTDI}_{\mathrm{vol}} \times \text { Conversion Factor. }
$$

\section{Statistical Analysis}

For statistical analysis, dedicated software (SPSS, Version 19; IBM Armonk, New York) was used. A $P$ value $<.05$ was significant for all tests. Data sphericity was assessed with the Greenhouse-Geisser and the Huynh-Feldt methods. Continuous variables were expressed as means \pm SDs. Quantitative image analysis was evaluated by using repeated measures of ANOVA. Statistical analysis of qualitative image analysis was performed by using the nonparametric Wilcoxon test for the intraindividual comparison.

The interobserver acceptance of subjective image analysis was expressed by Cohen weighted analysis: $\kappa<0.30$ indicated slight agreement; $\kappa=0.3-0.7$, moderate agreement; $\kappa>0.7$, good agreement.

\section{RESULTS}

All examinations were performed without any complications. No examinations 


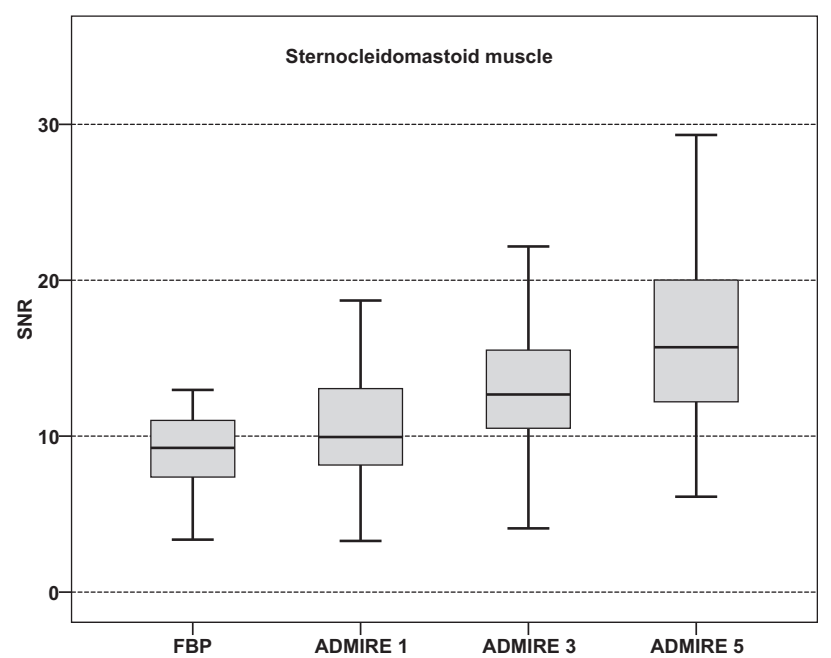

FIG 2. Boxplot graphs show comparison of signal-to-noise ratios of the sternocleidomastoid muscle. SNR was significantly higher for ADMIRE compared with FBP with the highest results observed for ADMIRE strength level 5 (all, $P<.001$ ). Significant differences were also shown within ADMIRE strength levels (all, $P<.001$ ).

were excluded because of severe motion or metal artifacts. Due to submandibular gland removal, evaluation of the submandibular gland was not possible in 2 cases.

The mean CT dose index volume was $6.57 \pm 0.75 \mathrm{mGy}$. The mean dose-length product was $174.4 \pm 31.3 \mathrm{mGy} \times \mathrm{cm}$, and the mean calculated effective dose was $0.89 \pm 0.16 \mathrm{mSv}$. The average effective diameter was $15.8 \pm 1.9 \mathrm{~cm}$, and the mean effective tube current was $227.0 \pm 26.1 \mathrm{mAs}$. The calculated average sizespecific dose estimate was $13.59 \pm 1.23 \mathrm{mGy}$.

\section{Objective Image Analysis}

Attenuation of the sternocleidomastoid muscle (all, $P>.405$ ) and submandibular gland (all $P>.245$ ) in FBP and all ADMIRE strength levels did not differ significantly. Image noise of the sternocleidomastoid muscle was significantly reduced by using ADMIRE compared with FBP with the lowest noise observed in ADMIRE 5 (all, $P<.001$ ). Image noise of the subscapularis muscle, representing noise levels in the lower part of the neck, was increased compared with noise of the sternocleidomastoid muscle, representing the upper part of the neck, within each reconstruction method (all, $P<.001$ ). Attenuation and image noise measurements are summarized in Table 1. Figure 1 illustrates a comparison of image noise between FBP and ADMIRE 1, 3, and 5 of the sternocleidomastoid muscle and subscapularis muscle.

SNR calculations are summarized in Table 2. The sternocleidomastoid muscle SNR was significantly higher for all reconstructed ADMIRE strength levels compared with FBP, with the best results in ADMIRE 5 and significant differences within all ADMIRE strength levels (all, $P<.001$ ). Comparisons of the sternocleidomastoid muscle SNR are illustrated in Fig 2.

Sternocleidomastoid muscle-fat CNR was significantly higher in ADMIRE 3 and 5 compared with FBP and ADMIRE 1, with the highest CNR in ADMIRE 5 (all, $P<.001$ ). Sternocleidomastoid muscle-fat CNR in FBP was slightly higher than that in ADMIRE $1(P=.256)$. Submandibular gland-fat CNR was highest in ADMIRE 5 compared with FBP and ADMIRE 1 and $3(P<.001)$.
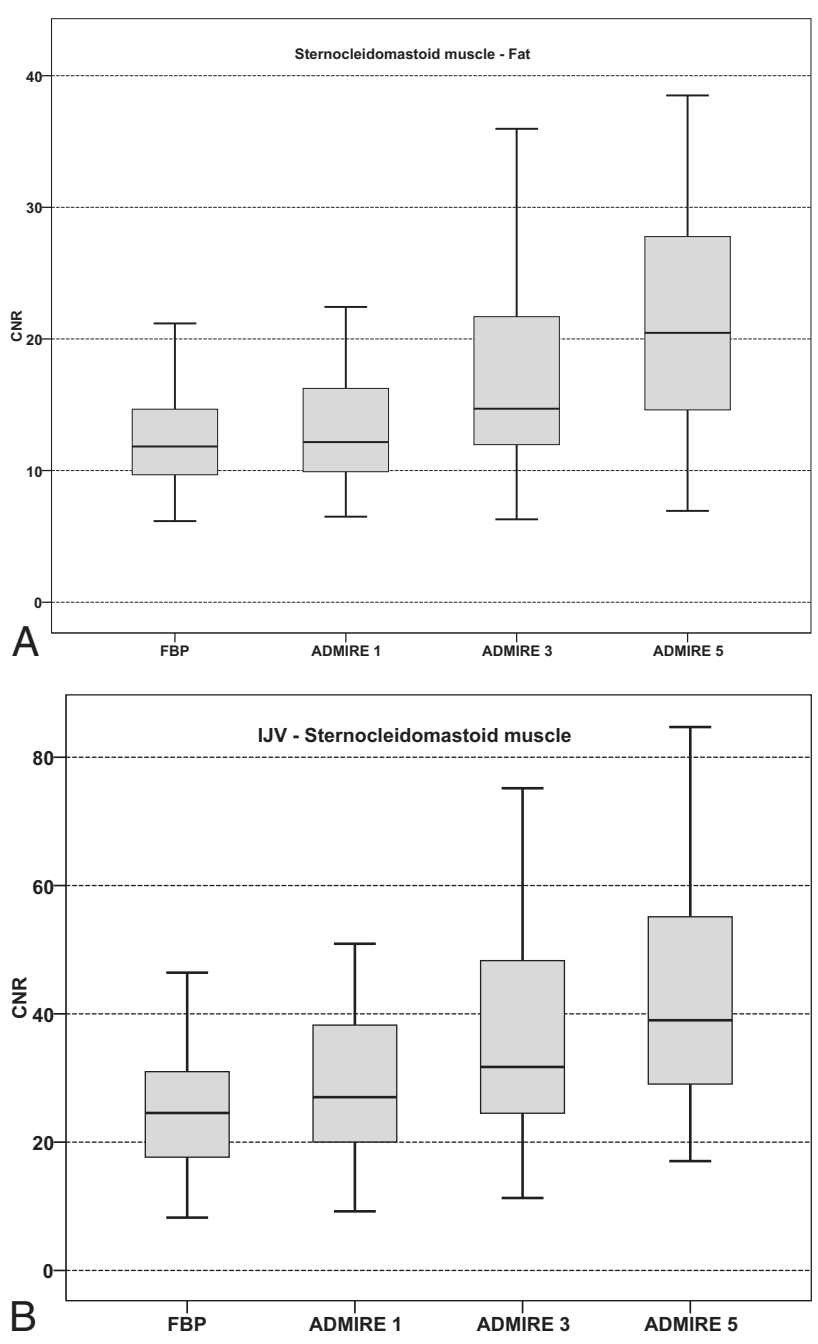

FIG 3. Boxplot graphs show contrast-to-noise ratios of sternocleidomastoid muscle-to-fat $(A)$ with significantly higher results in ADMIRE strength levels 3 and 5 compared with FBP and ADMIRE $1(P<.001)$, while internal jugular vein-sternocleidomastoid muscle CNR $(B)$ is significantly increased in all ADMIRE strength levels compared with FBP (all, $P<.001$ ).

Submandibular gland-fat CNR in FBP was nonsignificantly higher compared with ADMIRE $1(P=.430)$ but was significantly lower compared with ADMIRE $3(P=.004)$. Internal jugular vein-sternocleidomastoid CNR was significantly higher in all ADMIRE strength levels compared with FBP, with the highest CNR in ADMIRE 5 and significant differences within the ADMIRE strength levels (all, $P<.001$ ). Results of CNR calculations are summarized in Table 2, and comparisons of CNR calculations are shown in Fig 3. Figure 4 shows axial images of a bilateral T2 glottic larynx cancer by using FBP and ADMIRE 1, 3, and 5.

\section{Subjective Image Analysis}

Results from subjective ratings including interobserver agreement are summarized in Table 3. Overall image quality was voted best in ADMIRE 5, with moderate interobserver agreement and slightly but not significantly better results compared with ADMIRE $3(P=.088)$. Both ADMIRE 3 and 5 were voted significantly higher than FBP and ADMIRE $1(P<.001)$. ADMIRE 1 was rated slightly better than FBP $(P=.109)$. 

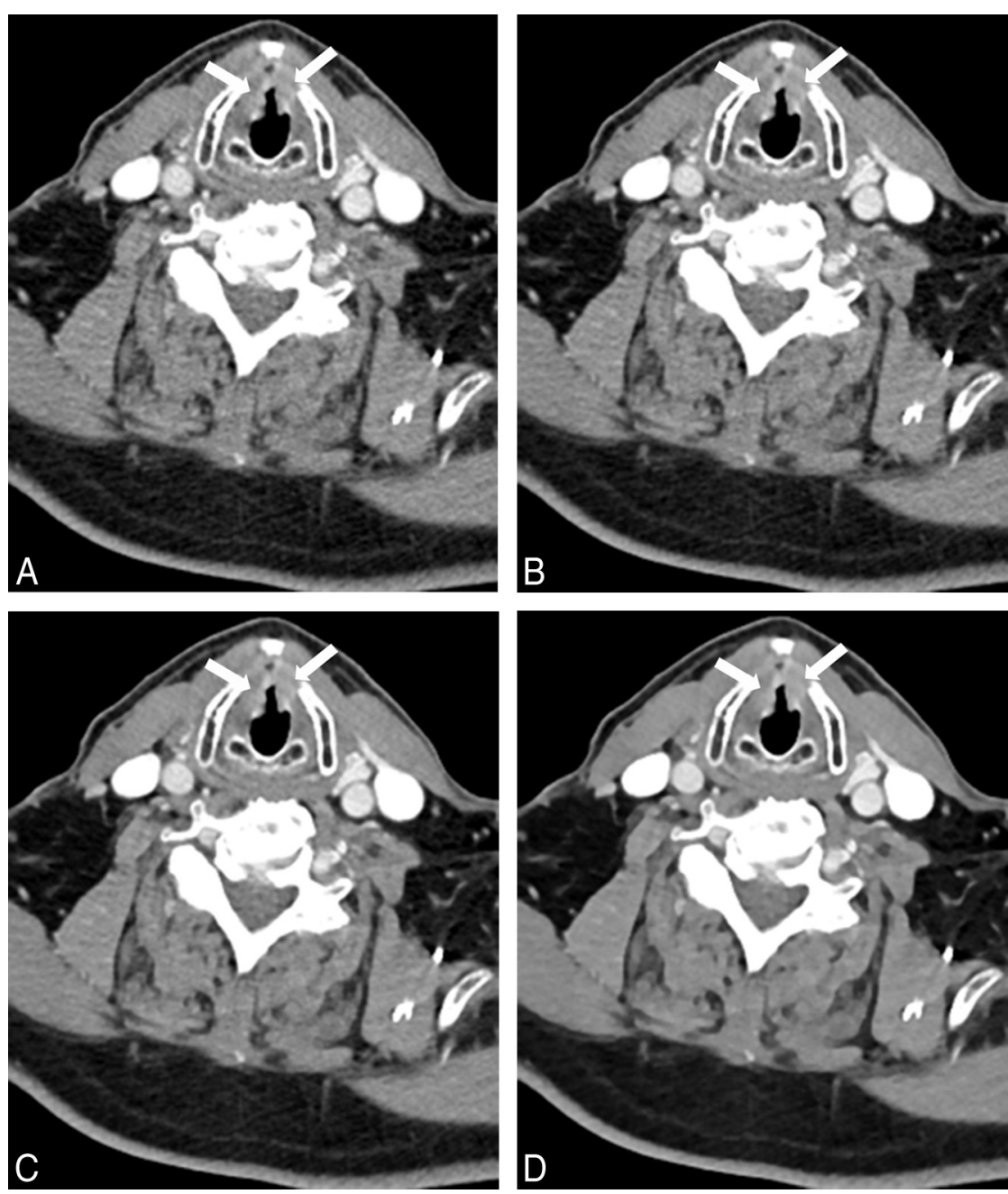

FIG 4. Images of a 46-year-old male patient examined with a low tube voltage of $90 \mathrm{kV}$ on 192-section DSCT (window settings: width, $400 \mathrm{HU}$; level, $80 \mathrm{HU}$ ). Images were reconstructed by using filtered back-projection $(A)$ and advanced modeled iterative reconstruction with strength levels $1(B), 3(C)$, and $5(D)$. Axial images show histologically proved bilateral T2 squamous cell carcinoma of the glottic larynx (arrows). Image noise was highest by using FBP $(A)$. The higher ADMIRE strength levels show consistently lower image noise $(B-D)$. The internal jugular veinsternocleidomastoid muscle CNR is highest by using ADMIRE $5(D)$. Delineation of smaller structures was considered good by both observers in all images.

Image noise of all ADMIRE strength levels was voted significantly better than FBP with significant differences within ADMIRE strength levels and the highest ratings for ADMIRE 5 (all, $P<.005$ ).

Delineation of smaller structures was voted significantly better in all ADMIRE strength levels compared with FBP (all, $P \leq .001$ ) without significant differences among the ADMIRE levels (ADMIRE 1 versus $3, P=.071$; ADMIRE 1 versus $5, P=.285$; ADMIRE 3 versus $5, P=.316)$. Interobserver agreement of the delineation of smaller structures was moderate to good $(\kappa=$ $0.50-0.78)$. Figure 5 shows axial images of a bilateral T4 glottic larynx cancer.

Image contrast was rated highest in ADMIRE 3 and $5(P=$ .769). Both ADMIRE 3 and 5 were voted significantly better than FBP and ADMIRE 1 (all, $P<.001$ ). FBP was voted slightly better than ADMIRE $1(P=.157)$. Global interobserver agreement was $\operatorname{good}(\kappa=0.75)$.

\section{DISCUSSION}

The results of our study indicate that ADMIRE improves image quality for low-tube-voltage contrast-enhanced neck CT compared with FBP. Image noise was reduced significantly, and SNR and CNR were increased with ADMIRE. Objective image quality peaked with ADMIRE 5, while subjective image quality was superior in ADMIRE 3 and 5. Our protocol may be beneficial in clinical routine for reducing radiation exposure in patients undergoing neck CT without impairing image quality.

Repetitive use of CT may result in a substantial radiation exposure with a generally rising cumulative radiation dose in the population that may potentially increase the risk for radiation-induced carcinogenesis. ${ }^{21,22}$ Tube-voltage reduction is one of several opportunities to reduce radiation exposure substantially in contrast-enhanced neck CT. ${ }^{11,23}$ A potential drawback of a reduced tube voltage is an increase in image noise. ${ }^{4,5,11,23,24}$ Due to increased image noise, $70 \mathrm{kV}$ (peak) neck CT has compromised image quality in the lower part of the neck. ${ }^{23}$ As a consequence of limited diagnostic acceptability, May et $\mathrm{al}^{11}$ excluded $80-\mathrm{kVp}$ acquisitions by using automated tube voltage adaptation in contrast-enhanced neck CT in combination with FBP on a second-generation 128-section DSCT. Nevertheless, an average radiation dose reduction of $8 \%-9 \%$ was measured in automated tube voltage adaptation ranging from 100 to $140 \mathrm{kVp} .{ }^{11}$ In contrast and despite an increased image noise, prior studies investigating $80-\mathrm{kVp}$ contrast-enhanced neck CT in combination with FBP on the same second-generation DSCT reported an increased tumor delineation and good diagnostic accuracy of benign and malignant pathologies. ${ }^{4,5,24}$ The average size-specific dose estimate in our study was $13.59 \pm 1.23 \mathrm{mGy}$, which corresponds to a dose reduction of approximately $35.4 \%$ compared with standard $120 \mathrm{kVp}$.

Iterative reconstruction has quickly become a standard feature on modern CT scanners, providing image noise reduction. ${ }^{18,25}$ Gaddikeri et al ${ }^{12}$ recently reported a substantial image noise reduction and increased SNR and CNR in 140-kV contrast-enhanced neck CT by using model-based iterative reconstruction compared with FBP. Nevertheless, image acquisition with 100 or $120 \mathrm{kV}$ is more commonly encountered due to a lower radiation dose and improved iodine attenuation. Prior studies have reported that the combination of a reduced tube voltage and an iterative reconstruction algorithm may allow substantial radia- 

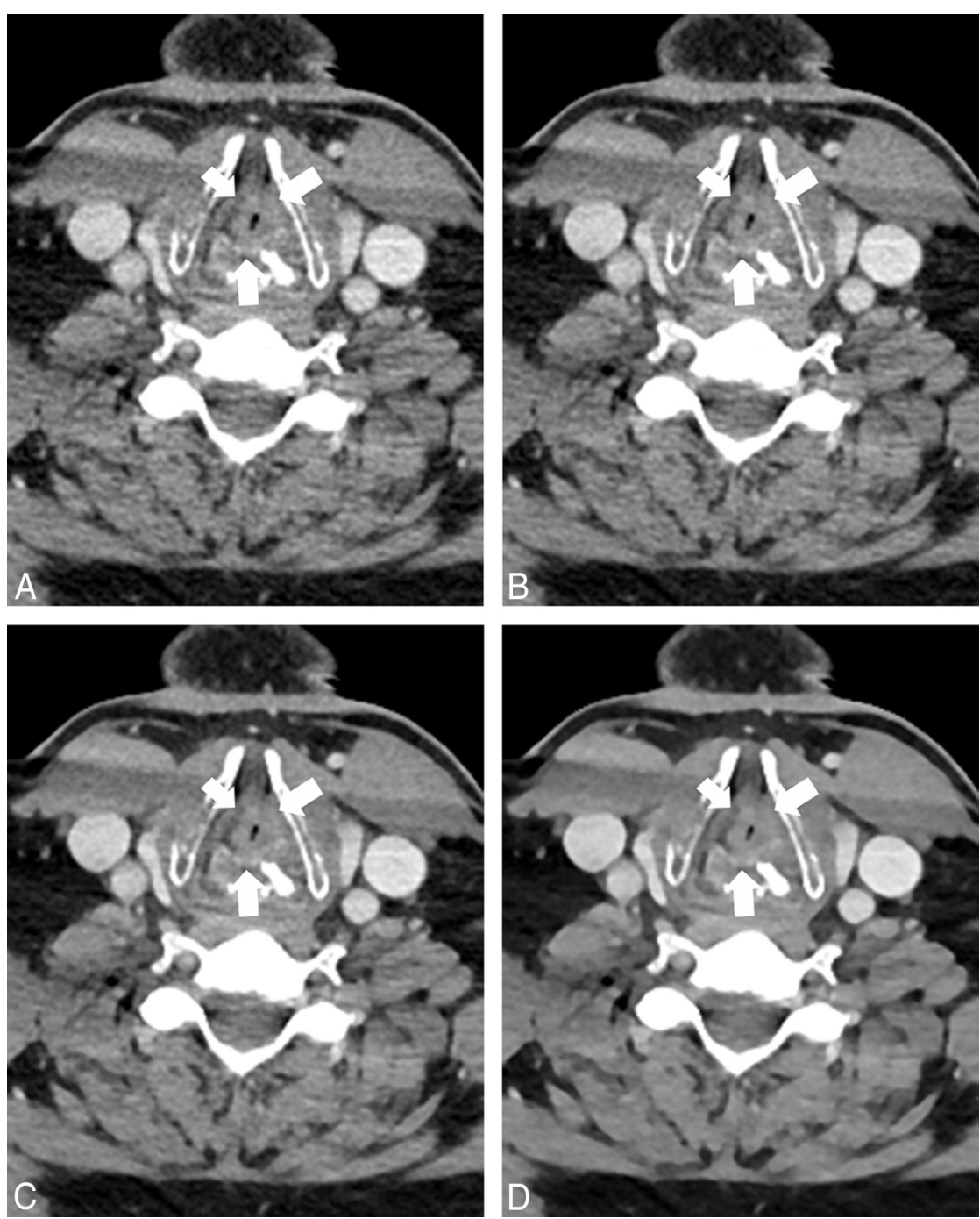

FIG 5. A 58-year-old male patient with sudden dyspnea. CT was performed with a tube voltage of $90 \mathrm{kV}$ (window settings: width, $400 \mathrm{HU}$; level, $80 \mathrm{HU}$ ). Images were reconstructed by using filtered back-projection $(A)$ and advanced modeled iterative reconstruction with strength levels $1(B), 3(C)$, and $5(D)$. Images show histologically proved bilateral T4 laryngeal squamous cell carcinoma (arrows). The tumor reaches to the left thyroid cartilage but is separated from the right thyroid cartilage by a thin fat line. Image noise was lower in ADMIRE compared with FBP with the lowest image noise in ADMIRE $5(D)$. Streak artifacts in the sternocleidomastoid muscle on both sides are visible in all images due to the shoulder region in the lower part of the neck.

tion dose reduction without impairment of image quality. ${ }^{12-14,26}$ Third-generation DSCT has improved tube efficiency at lower tube voltages and is equipped with a model-based ADMIRE algorithm to provide adequate image quality in low-tube-voltage acquisitions. ADMIRE has been shown to provide an improved 3D regularization process, which results in better noise reduction in the image domain. ${ }^{14}$ Furthermore, fewer noise streaks and better artifact suppression are facilitated by detailed modeling in the projection data domain. Initial results were reported by Gordic et $\mathrm{al}^{14}$ who observed reduced image noise with higher ADMIRE strength levels in 90-120 kV abdominal CT. Furthermore, SNR and CNR were significantly increased in all measured abdominal soft-tissue structures with the best results for ADMIRE strength levels 4 and 5. In our study, similar results were observed for 90-kV acquisitions: Attenuation of anatomic structures of the neck was constant between FBP and ADMIRE, while objectively and subjectively measured image noise was significantly reduced with ADMIRE compared with FBP, with lowest noise observed in ADMIRE 5. This result is in accordance with those reported by Gordic et $\mathrm{al}^{14}$ measuring the lowest image noise in $90-\mathrm{kV}$ abdominal CT for ADMIRE 5. Solomon et $\mathrm{al}^{18}$ reported a substantial radiation dose reduction in ADMIRE compared with FBP while preserving detectability in a low-contrast acquisition in a phantom study.

Image noise in the lower part of the neck is significantly higher compared with the upper part due to superimposition of the shoulder region. ${ }^{12,23}$ Gnannt et $\mathrm{al}^{23}$ reported worse visualization in the lower third of the neck in a $70-\mathrm{kVp}$ acquisition when combined with FBP on a 64-section CT. Our results demonstrate that ADMIRE is unable to compensate for image noise differences between the upper and lower parts of the neck, but it significantly reduces image noise in both upper and lower neck space with the lowest noise observed with ADMIRE 5. Similar findings were reported by Gaddikeri et $\mathrm{al}^{12}$ for modelbased iterative reconstruction compared with FBP in $140-\mathrm{kVp}$ contrast-enhanced neck CT. Thus, the lower part of the neck remains a critical area to evaluate when applying dose-saving scan protocols, and application of these protocols should be considered on the basis of the indication for imaging.

In line with previously published studies, SNR and CNR were increased in ADMIRE with the highest results in ADMIRE 5. ${ }^{14,18}$ Nevertheless, we also observed higher variations in both parameters with ADMIRE 5. Because attenuation values of all softtissue structures were constant in FBP and ADMIRE, the cause of the increase in SNR and CNR is the reduction of image noise. A further reduction of tube voltage may result in an increase of attenuation of ionic soft-tissue structures with the additional increase of SNR. However, the performance of ADMIRE reconstruction in such imaging protocols should be investigated in future studies.

Subjective image results were also favorable for higher ADMIRE levels, similar to the objectively measured results. Image noise was rated best in ADMIRE 5 compared with FBP and ADMIRE 1 and 3. Delineation of smaller neck structures was voted better in all ADMIRE strength levels compared with FBP. Slight differences within ADMIRE strength levels did not reach statistical significance. Image contrast and overall image quality were voted excellent in both ADMIRE 3 and 5, with significantly 
better ratings compared with FBP and ADMIRE 1. Although ADMIRE 5 has been thought to provide an artificially smoothed image impression in prior studies, both image contrast and overall image quality were rated slightly but not significantly better in ADMIRE 5 compared with ADMIRE 3.

Due to the low image noise and the excellent image quality in $90-\mathrm{kVp}$ acquisitions in combination with ADMIRE in contrastenhanced neck CT in our study, which is the current standard protocol at our institution, we suggest additional investigations on the diagnostic accuracy of benign and malignant neck pathologies in $90-\mathrm{kVp}$ acquisitions. Furthermore, the feasibility of tube voltage reduction to 80 and $70 \mathrm{kVp}$ with respect to the patient's anatomy, such as a short neck, should be further evaluated.

Some limitations of our study need to be addressed. First, we performed a comparison of ADMIRE versus FBP. Together with the implementation of ADMIRE, a new third-generation 192section DSCT was implemented in our system. An additional comparison of different generations of iterative reconstruction techniques and DSCT systems would have been favorable. However, an intraindividual comparison in follow-up examinations would have been influenced by hardware differences between second-generation 128-section and third-generation 192-section DSCT and additional differences in standard scan protocol parameters, including tube voltage and potential. Second, while a cohort of 64 patients is sufficient for an initial feasibility study, additional evaluation in a larger, more diversified cohort is necessary. Third, we did not investigate the combination of a standard $120-\mathrm{kV}$ acquisition with ADMIRE, because $90-\mathrm{kV}$ is our current protocol for contrast-enhanced neck CT by using 192section DSCT. Furthermore, we did not focus on specific neck pathologies separately but evaluated subsequent patients with clinical indications for head and neck CT. Further studies should focus on specific neck pathologies, including visualization of squamous cell carcinoma or cervical lymphoma staging and follow-up, and inflammation of soft-tissue structures. In addition, low-tube-voltage CT may result in suboptimal image quality in patients with a short neck with a wide diameter. Our technique should be re-evaluated in this specific patient population for its impact on the detection of laryngeal or hypopharyngeal cancer. Finally, we did not evaluate differences in the diagnostic performance of the various ADMIRE reconstruction levels. The initial goal of our study was to demonstrate the feasibility of the lowtube-voltage $90-\mathrm{kV}$ acquisition and to find the best reconstruction parameters to achieve optimal image quality by applying ADMIRE to reduce image noise caused by CT scanning with a low tube voltage. Performing multireader evaluations of 3 different ADMIRE reconstructions in each patient to assess diagnostic performance would have led to recall bias. Nevertheless, we plan to directly compare low-tube-voltage ADMIRE reconstructions with standard 120-kV FBP scans in the near future for the diagnostic performance for the detection of head and neck malignancy.

\section{CONCLUSIONS}

Our results demonstrate that low-tube-voltage acquisitions in combination with ADMIRE significantly reduce image noise and increase SNR and CNR in contrast-enhanced 192-section neck
CT. Objective image quality peaked by using ADMIRE 5, while ADMIRE 3 and 5 showed the best subjective image quality. Dosesaving low-tube-voltage $90-\mathrm{kVp}$ contrast-enhanced neck CT in combination with ADMIRE strength levels 3 or 5 may be routinely applied to enhance image quality and reduce the radiation dose in clinical routine.

Disclosures: Ralf W. Bauer-UNRELATED: Payment for Lectures (including service on Speakers Bureaus): Siemens, Comments: Speakers Bureau, CT division. J. Matthias Kerl-UNRELATED: Payment for Lectures (including service on Speakers Bureaus): Siemens, Comments: Speakers Bureau, CT division.

\section{REFERENCES}

1. Dammann F, Bootz F, Cohnen M, et al. Diagnostic imaging modalities in head and neck disease. Dtsch Arztebl Int 2014;111:417-23 CrossRef Medline

2. Gonzalez-Beicos A, Nunez D. Imaging of acute head and neck infections. Radiol Clin North Am 2012;50:73-83 CrossRef Medline

3. Griauzde J, Srinivasan A. Imaging of vascular lesions of the head and neck. Radiol Clin North Am 2015;53:197-213 CrossRef Medline

4. Scholtz JE, Kaup M, Kraft J, et al. Objective and subjective image quality of primary and recurrent squamous cell carcinoma on head and neck low-tube-voltage $80-\mathrm{kVp}$ computed tomography. Neuroradiology 2015;57:645-51 CrossRef Medline

5. Wichmann JL, Kraft J, Nöske EM, et al. Low-tube-voltage 80-kVp neck CT: evaluation of diagnostic accuracy and interobserver agreement. AJNR Am J Neuroradiol 2014;35:2376-81 CrossRef Medline

6. Schulz B, Potente S, Zangos S, et al. Ultra-low dose dual-source highpitch computed tomography of the paranasal sinus: diagnostic sensitivity and radiation dose. Acta Radiol 2012;53:435-40 CrossRef Medline

7. Greess $\mathrm{H}$, Wolf $\mathrm{H}$, Baum U, et al. Dose reduction in computed tomography by attenuation-based on-line modulation of tube current: evaluation of six anatomical regions. Eur Radiol 2000;10: 391-94 CrossRef Medline

8. Papadakis AE, Perisinakis K, Damilakis J. Automatic exposure control in pediatric and adult multidetector CT examinations: a phantom study on dose reduction and image quality. Med Phys 2008;35: 4567-76 CrossRef Medline

9. Söderberg M, Gunnarsson M. Automatic exposure control in computed tomography-an evaluation of systems from different manufacturers. Acta Radiol 2010;51:625-34 CrossRef Medline

10. Lurz M, Lell MM, Wuest W, et al. Automated tube voltage selection in thoracoabdominal computed tomography at high pitch using a third-generation dual-source scanner: image quality and radiation dose performance. Invest Radiol 2015;50:352-60 CrossRef Medline

11. May MS, Kramer MR, Eller A, et al. Automated tube voltage adaptation in head and neck computed tomography between 120 and $100 \mathrm{kV}$ : effects on image quality and radiation dose. Neuroradiology 2014;56:797-803 CrossRef Medline

12. Gaddikeri S, Andre JB, Benjert J, et al. Impact of model-based iterative reconstruction on image quality of contrast-enhanced neck CT. AJNR Am J Neuroradiol 2015;36:391-96 CrossRef Medline

13. Bodelle B, Klein E, Naguib NN, et al. Acute intracranial hemorrhage in CT: benefits of sinogram-affirmed iterative reconstruction techniques. AJNR Am J Neuroradiol 2014;35:445-49 CrossRef Medline

14. Gordic S, Desbiolles L, Stolzmann P, et al. Advanced modelled iterative reconstruction for abdominal CT: qualitative and quantitative evaluation. Clin Radiol 2014;69:e497-504 CrossRef Medline

15. Korn A, Fenchel $M$, Bender B, et al. Iterative reconstruction in head $\mathrm{CT}$ : image quality of routine and low-dose protocols in comparison with standard filtered back-projection. AJNR Am J Neuroradiol 2012;33:218-24 CrossRef Medline

16. Curtis JR. Computed tomography shielding methods: a literature review. Radiol Technol 2010;81:428-36 Medline

17. Ramirez-Giraldo J, Primak A, Grant K, et al. Radiation dose optimi- 
zation technologies in multidetector computed tomography: a review. Med Phys 2014;2:420-30

18. Solomon J, Mileto A, Ramirez-Giraldo JC, et al. Diagnostic performance of an advanced modeled iterative reconstruction algorithm for low-contrast detectability with a third-generation dual-source multidetector CT scanner: potential for radiation dose reduction in a multireader study. Radiology 2015;275:735-45 CrossRef Medline

19. Gordic S, Morsbach F, Schmidt B, et al. Ultralow-dose chest computed tomography for pulmonary nodule detection: first performance evaluation of single energy scanning with spectral shaping. Invest Radiol 2014;49:465-73 CrossRef Medline

20. Computed Tomography Subcommittee Task Group \#204. Size specific dose estimates (SSDE) in pediatric and adult body CT examinations. American Association of Physicists in Medicine Reports. 2011. http://www.aapm.org/pubs/reports/default.asp. Accessed June 7, 2015

21. Berrington de González A, Mahesh M, Kim KP, et al. Projected cancer risks from computed tomographic scans performed in the
United States in 2007. Arch Intern Med 2009;169:2071-77 CrossRef Medline

22. Brenner DJ, Hall EJ. Computed tomography: an increasing source of radiation exposure. $N$ Engl J Med 2007;357:2277-84 CrossRef Medline

23. Gnannt R, Winklehner A, Goetti R, et al. Low kilovoltage CT of the neck with $70 \mathrm{kVp}$ : comparison with a standard protocol. AJNR AmJ Neuroradiol 2012;33:1014-19 CrossRef Medline

24. Scholtz JE, Hüsers K, Kaup M, et al. Evaluation of image quality and dose reduction of $80 \mathrm{kVp}$ neck computed tomography in patients with suspected peritonsillar abscess. Clin Radiol 2015;70:e67-73 CrossRef Medline

25. Beister M, Kolditz D, Kalender WA. Iterative reconstruction methods in x-ray CT. Phys Med 2012;28:94-108 CrossRef Medline

26. May MS, Wüst W, Brand M, et al. Dose reduction in abdominal computed tomography: intraindividual comparison of image quality of full-dose standard and half-dose iterative reconstructions with dual-source computed tomography. Invest Radiol 2011;46: 465-70 CrossRef Medline 